Wilfrid Laurier University

Scholars Commons @ Laurier

Physics and Computer Science Faculty

Publications

Physics and Computer Science

1993

\title{
Dynamical Properties of Josephson Junctions Coupled by a Transmission Line
}

\author{
H.J.T. Smith \\ University of Waterloo \\ James A. Blackburn \\ Wilfrid Laurier University, jabjabjab@cogeco.ca
}

Niels Grønbech-Jensen

Los Alamos National Laboratory

Follow this and additional works at: https://scholars.wlu.ca/phys_faculty

\section{Recommended Citation}

Smith, H.J.T.; Blackburn, James A.; and Grønbech-Jensen, Niels, "Dynamical Properties of Josephson Junctions Coupled by a Transmission Line" (1993). Physics and Computer Science Faculty Publications. 42.

https://scholars.wlu.ca/phys_faculty/42

This Article is brought to you for free and open access by the Physics and Computer Science at Scholars Commons @ Laurier. It has been accepted for inclusion in Physics and Computer Science Faculty Publications by an authorized administrator of Scholars Commons @ Laurier. For more information, please contact scholarscommons@wlu.ca. 


\title{
Dynamical properties of Josephson junctlons coupled by a transmission line
}

\author{
H. J. T. Smith \\ Department of Physics, University of Waterloo, Waterloo, Ontario N2L3G1, Canada \\ James A. Blackburn \\ Department of Physics and Computing, Wilfrid Laurier University, Waterloo, Ontario N2L3C5, Canada
}

Niels Gronbech-Jensen

Theoretical Division, Los Alamos National Laboratory, Los Alamos, New Mexico 87545

(Received 19 April 1993; accepted for publication 1 July 1993)

\begin{abstract}
A system composed of two Josephson junctions connected by a transmission line has been studied by means of electronic analog simulation. Under external current bias, the resistive component of the coupling induces frequency locking between the two junctions at commensurate ratios. The resonant modes of the transmission line give rise to steps in the $I-V$ characteristics of the system.
\end{abstract}

\section{INTRODUCTION}

Josephson junctions have been of considerable interest for many years both because of their potential practical applications in superconducting electronics, and because the nonlinear nature of their dynamics gives rise to various phenomena such as chaos. Although only a few commercial products based on superconducting devices have emerged, research is continuing in new areas such as analog-digital converters and digital logic. ${ }^{1}$ Circuits assembled from Josephson junctions are "wired" via thin-film interconnects which, at the high frequencies involved, act as striplines. ${ }^{2-4}$

In a previous paper, ${ }^{5}$ we studied the dynamics of a noncapacitive Josephson junction coupled to a resistively terminated transmission line. The present work is a generalization to the case where the termination is a second Josephson junction, or equivalently, where two junctions are coupled by a transmission line.

The coupling of two nonlinear devices was first reported by Huygens who observed that when two pendula were coupled by mounting them adjacent to each other on the same beam the periods became synchronized. A general review of synchronization is given by Blekhman. ${ }^{6}$ Synchronization can occur between nonlinear devices because the resonant frequency of each device is a function of the amplitude of the oscillation of that device and, when coupled together, the oscillators can adjust their amplitudes and hence their resonant frequencies until these resonant frequencies are either identical or commensurate. On the other hand, two coupled linear devices cannot synchronize, but will instead give rise to such phenomena as beats.

The possible modes of motion of an isolated junction consist of oscillations of the phase angle about either a fixed value or a steadily rotating value. When a pair of junctions can interact, the two junction frequencies would be expected to form a commensurate ratio regardless of the mode of motion. In the case to be considered here, the coupling is provided by a transmission line with its own characteristic resonances; hence the strength of the coupling will generally be frequency dependent and will be further influenced by resonance broadening due to damping in the line.

As in our earlier work, ${ }^{5}$ electronic analogs of the transmission line and Josephson junctions are used to model the system. This experimental approach is extremely fast compared to direct computational solutions obtained from numerical simulations of the complete set of coupled differential equations, although there is inevitably some trade-off in precision. To avoid confusion in the discussions to follow, we shall adopt the convention of referring to data obtained from electronic analogs as "experimental," and data generated from numerical solutions as "simulation."

\section{RESISTOR COUPLING}

It is instructive to first consider the situation when the distributed reactances are deleted from the transmission line, leaving only the simple coupling indicated in Fig. 1. In so doing, we are temporarily setting aside the resonant attributes of the transmission line so as to focus on the contribution of the line resistance alone to the locking of the junction oscillations. Surprisingly, this arrangement of parallel shunt coupling does not appear to have been considered before, although series connected weak links with resistive $^{7-9}$ and reactive ${ }^{10-12}$ shunts have been the subject of many reports.

An electronic analog of this configuration is depicted schematically in Fig. 2. The operation of the two Josephson junction modules (in boxes) has been discussed in previous publications. ${ }^{5,13}$ The central op-amp with the $R_{f}$ feedback resistor serves to impose Kirchhoff's law:

$$
V_{J A}=I_{B} R_{\text {tot }}+V_{J B} \text {. }
$$

The equivalence of the coupling are $R_{\mathrm{tot}}=R^{2} / R_{f}$. The complete transmission line, to be discussed in the next section, is made up of 71 discrete " $T$ " segments, each containing a series resistance $R_{s}=152 \Omega$; hence $R_{\text {tot }}=71 \times 152=10.79 \mathrm{~K}$. Therefore since $R$ was chosen to be $39 \mathrm{~K}, R_{f}=141 \mathrm{~K}$.

The time-averaged voltage across each of the junctions was measured as a function of the input bias voltage $V_{\text {bias }}$. 


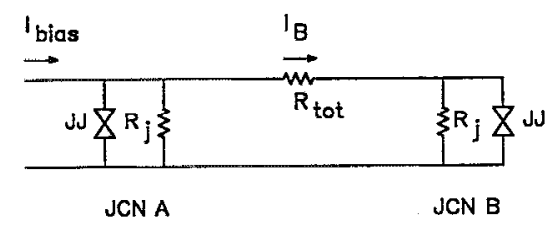

FIG. 1. Two Josephson junctions coupled via a resistor.

The corresponding input bias current as depicted in Fig. 1 is thus $I_{\text {bias }}=V_{\text {bias }} / R$. These measurements were performed using a PC-based data acquisition system consisting of a programmable dc power supply for $V_{\text {bias }}(\mathrm{HP} 6632 \mathrm{~A})$ and digital voltmeters ( $\mathrm{HP} 3478 \mathrm{~A}$ ), all connected to the $\mathrm{PC}$ via the GPIB bus. The controlling software was written in Microsoft QuickBASIC 4.5. Each $I-V$ curve is composed of 1400 points, spaced at $5 \mathrm{mV}$ intervals in $V_{\text {bias }}$. The waveforms from the junctions were prefiltered so the voltmeters would see essentially only time-averaged values. Figure 3 displays the experimental results.

Input bias-junction voltage characteristics were also obtained from a numerical solution of the appropriate equations. Such a solution is comparatively easy in this resistively coupled case (as opposed to the general transmission line coupling to be treated in the next section). For the arrangement shown in Fig. 1, the equations are

$$
[1+\alpha] \frac{d \phi_{A}}{d t}-[\alpha] \frac{d \phi_{B}}{d t}+\sin \left(\phi_{A}\right)=\frac{I_{\mathrm{bias}}}{I_{c}}
$$

and

$$
[1+\alpha] \frac{d \phi_{B}}{d t}-[\alpha] \frac{d \phi_{A}}{d t}+\sin \left(\phi_{B}\right)=0,
$$

where $\phi_{A}$ and $\phi_{B}$ are the junction phases, $\alpha=R_{J} / R_{\text {tot }}$ ( $=2.32$ for this study), $I_{\text {bias }}$ is the input bias current, $I_{c}$ is the critical current (assumcd equal for the two junctions),

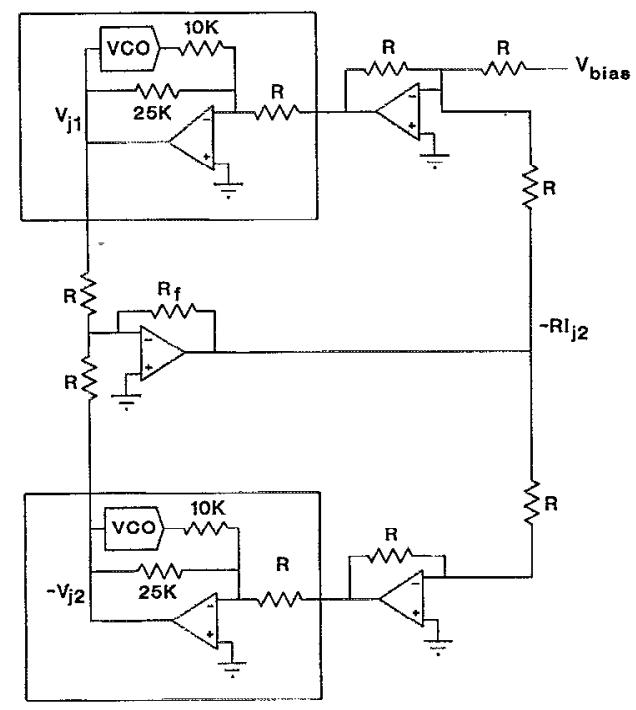

FIG. 2. Schematic of analog simulation circuit for resistively coupled Josephson junctions. The op-amps are type 741 and $R=39 \mathrm{~K}$.

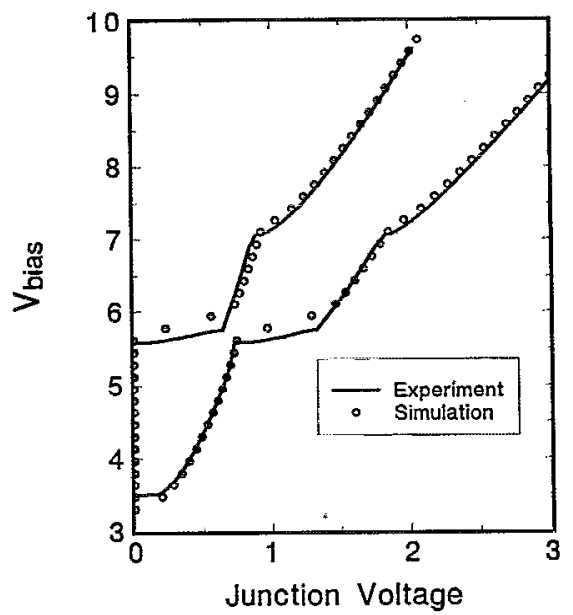

FIG. 3. Input bias (volts) vs time-averaged junction voltage obtained from the electronic analog. Upper characteristic: termination junction; lower characteristic: input junction. Circles are from numerical simulation.

and time is expressed in units of $\hbar /\left(2 e I_{c} R_{J}\right)$. The electronic Josephson analogs were characterized by $I_{c}=86 \mu \mathrm{A}$ and $R_{J}=25 \mathrm{k} \Omega$. The voltage-controlled oscillators ( $\mathrm{VCO}$ ) were designed to have $1000 \mathrm{~Hz}$ per volt (in fact they were within a few percent of this value). Because this is the analog counterpart of $2 e / h$, a unit of time in Eqs. (2) and (3) equals $\left(2 \pi \times 1000 \times I_{c} R_{J}\right)^{-1}=74 \mu$ s of electronic ana$\log$ time. In dimensionless time the usual Josephson voltage/phase relation is simply $d \phi / d t=V_{J} / I_{c} R_{J}$ and so an average $\langle d \phi / d t\rangle$ produced from the numerical simulation must be multiplied by 2.15 to yield an equivalent voltage for comparison with experiments.

Numerical solutions of Eqs. (2) and (3) were obtained using a time grid $\Delta t=0.01$. "dc" values $\langle d \phi / d t\rangle$ were evaluated by averaging over several thousand cycles of $d \phi / d t$. Results of these computations are shown as data points in Fig. 3. The very good agreement which is evident between simulation and experiment serves as a confirmation of the accuracy of the electronic circuit as an analog of the coupled Josephson junctions.

At low bias levels $3.5<V_{\text {bias }}<5.5$, the phase on the input junction is rotating thus resulting in nonzero $\left\langle d \phi_{A} / d t\right\rangle$, while the phase on the terminating junction oscillates such that $\left\langle d \phi_{B} / d t\right\rangle=0$. Typical waveforms from numerical simulations are shown in Fig. 4. Because the terminating junction carries less than $I_{c}$ in this bias range, it might seem that it should act simply as a superconducting short. However, a true short would clamp the voltage at zero, whereas the dynamic response of this load junction just guarantees that the average voltage is zero. Only in the limit of a junction with infinite critical current would $\phi_{B}$ be time independent.

The critical value of the bias current $I_{\mathrm{bc}}$ at which the load junction first enters the finite voltage state can be estimated with the following simple analysis. If all ac effects in the interaction between the two junctions are neglected, Eq. (2) can be written 


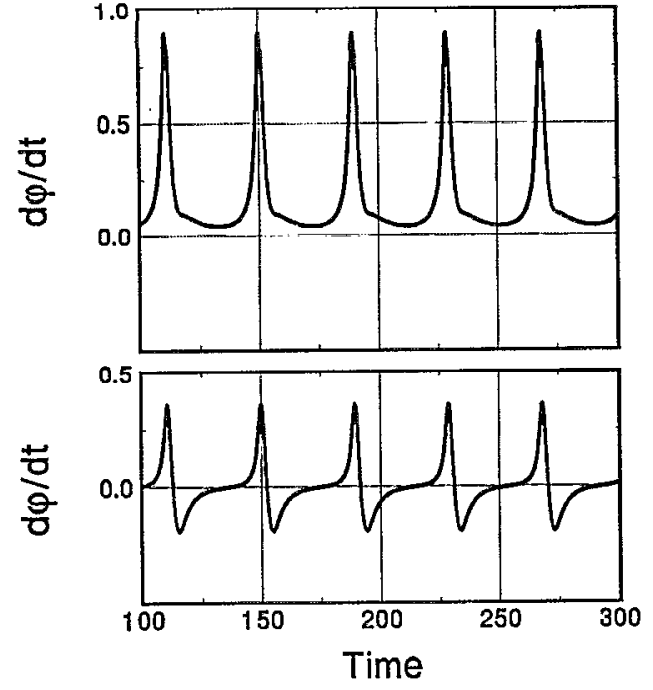

FIG. 4. Junction waveforms at $V_{\text {bias }}=3.8$ from numerical simulation. Upper trace: input junction; lower trace: terminating junction. An initial transient interval has been discarded.

$$
[1+\alpha] \frac{d \phi_{A}}{d t}+\sin \left(\phi_{A}\right)=\frac{I_{\text {bias }}}{I_{c}}
$$

and the average phase velocity of junction $A$ is then given by $^{14}$

$$
\left\langle\frac{d \phi_{A}}{d t}\right\rangle=\frac{1}{(1+\alpha)} \sqrt{\left(\frac{I_{\mathrm{hias}}}{I_{c}}\right)^{2}-1}
$$

For $1<I_{\mathrm{bias}} / I_{c}<I_{\mathrm{bc}} / I_{c}$, junction $B$ still maintains $\left\langle d \phi_{B} / d t\right\rangle=0$ and so Eq. (3) becomes

$$
-\alpha\left\langle\frac{d \phi_{A}}{d t}\right\rangle+\sin \left(\phi_{B}\right)=0
$$

From Eqs. (5) and (6), and noting that $\sin ^{2}\left(\phi_{B}\right)<1$, we obtain the critical bias current from the condition

$$
\frac{I_{\mathrm{bias}}}{I_{c}} \leqslant \frac{I_{\mathrm{bc}}}{I_{c}}=\sqrt{\left(\alpha^{-1}+1\right)^{2}+1} .
$$

For $\alpha=2.32$, the critical bias is then $V_{\text {bias }}=R I_{\mathrm{bc}}=5.8$. This value is quite close to the switching point $V_{\text {bias }} \approx 5.6 \mathrm{ob}-$ served in the numerical simulation data (Fig. 3).

Above $V_{\text {bias }} \approx 5.6$, the load junction receives enough current to force phase rotation. As a particular example, consider the case for $V_{\text {bias }}=8.3$. The junction phase velocities at this bias are shown in Fig. 5, where it can be seen that the voltage oscillations are locked. Both $d \phi_{A} / d t$ and $d \phi_{B} / d t$ have identical repeat intervals of 26.3 normalized time units. The essential nature of this locking is revealed by examining the corresponding phase motion as depicted in Fig. 6: during the repeat interval the phase on the load junction completes three revolutions while the phase on the input junction completes five revolutions. This will be denoted as 3:5 locking.

Computed power spectra of the $d \phi_{A} / d t$ and $d \phi_{B} / d t$ waveforms are plotted in Fig. 7. All the peaks are equally spaced along the frequency axis in increments of 0.038 ,
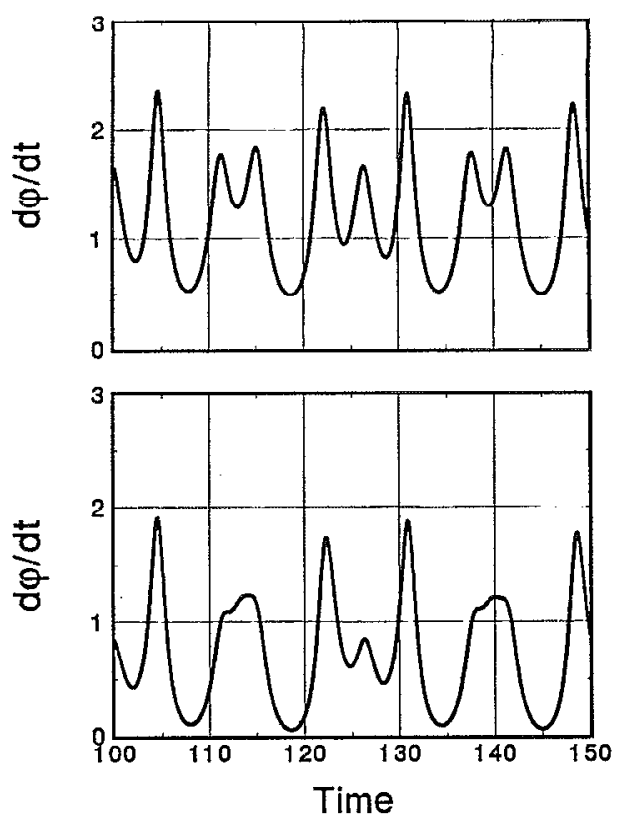

FIG. 5. Junction waveforms at $V_{\text {bias }}=8.3$ from numerical simulation. Upper trace: input junction; lower trace: terminating junction. An initial transient interval has been discarded.

which is simply the reciprocal of the previously mentioned repeat interval of 26.3. The complex voltage waveforms clearly contain two principal frequencies, $f_{A}$ and $f_{B}$, as well as various other components at frequencies determined by multiples of sums and differences of $f_{A}$ and $f_{B}$. This occurs because of the nonlinear mixing mediated by the junctions themselves.

For an isolated Josephson junction, $\omega=2 \mathrm{eV} / \hbar$, where $\omega$ is the angular frequency of the junction oscillation. In normalized units, this is expressed as $f=(2 \pi)^{-1}\left(V / I_{c} R_{J}\right)=(2 \pi)^{-1}(d \phi / d t)$. Careful examination of the data in Figs. 3 and 7 shows that the following relations are satisfied.

$$
f_{A}=\frac{1}{2 \pi}\left\langle\frac{d \phi_{A}}{d t}\right\rangle ; \quad f_{B}=\frac{1}{2 \pi}\left\langle\frac{d \phi_{B}}{d t}\right\rangle
$$

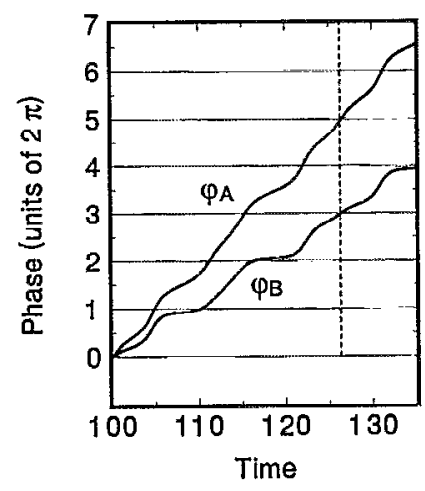

FIG. 6. Junction phases vs time (normalized units) obtained from numerical simulation. The dotted line marks the end of one repeat interval. An initial transient interval has been discarded. 

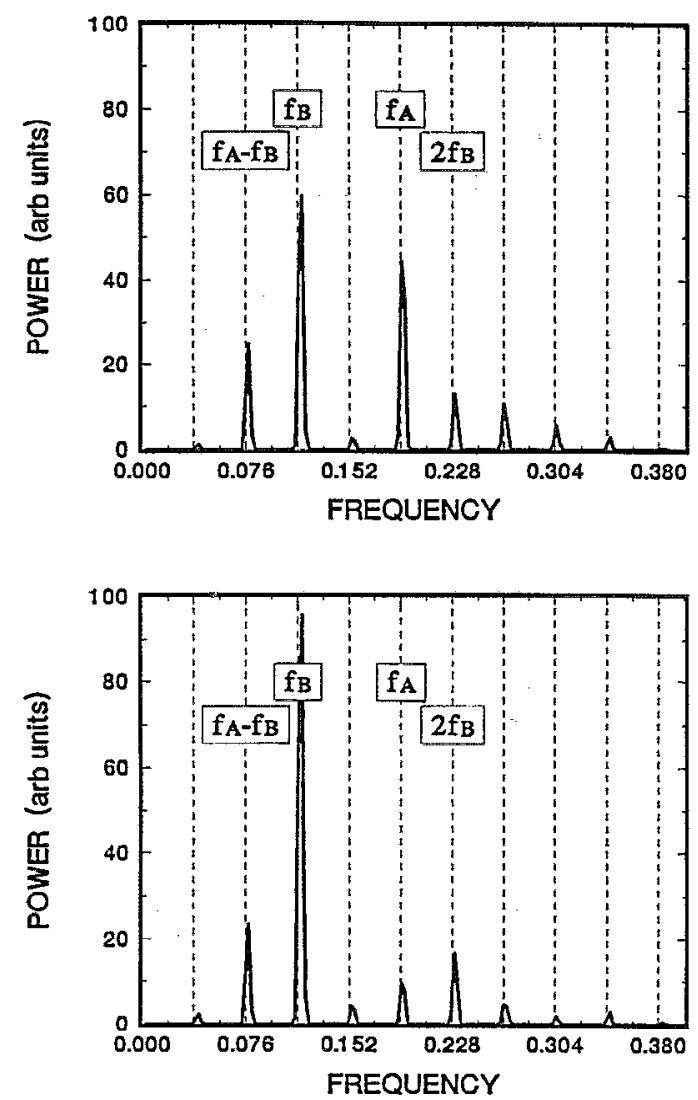

FIG. 7. Power spectra of junction waveforms at $V_{\text {bias }}=8.3$ obtained from numerical simulation. Upper: input junction; lower: termination junction. The abscissa is in normalized frequency units.

In other words, the two principal frequency components are determined by the average phase velocities (voltages).

The locking ratio was observed to increase as the bias was increased. It was, for example, 5:8 at $V_{\text {bias }}=9.0$. In each junction, the current is composed of a normal channel component $V / R_{J}$, and a supercurrent $I_{c} \sin (\phi)$. At high bias currents, because the supercurrent can never exceed $I_{c}$, most of the flow is through the normal channel. Ultimately, then, the combination of junction resistors and $R_{\text {tot }}$ just acts like a voltage divider and it would be expected that the ratio of the junction dc voltages, and hence the locking ratio, would approach the limiting value $R_{j} /\left(R_{j}+R_{\text {tot }}\right)=\alpha /(\alpha+1)=0.698$. Such a limit is consistent with the experimental results in Fig. 3, where the ratio of the two junction voltages has reached the value 0.628 at $V_{\text {bias }}=9.2$.

\section{TRANSMISSION LINE COUPLING}

We now turn to the more general situation, illustrated in Fig. 8, of a pair of Josephson junctions linked by a transmission line. The capacitive and inductive reactances which were eliminated in the preceding discussion, are now restored to the problem. For this case a lumped line consisting of 71 " $T$ " segments was modeled by a modified version of the circuit described in Ref. 5. Only the termination section [Ref. 5, Fig. 5(b)], which originally repre-

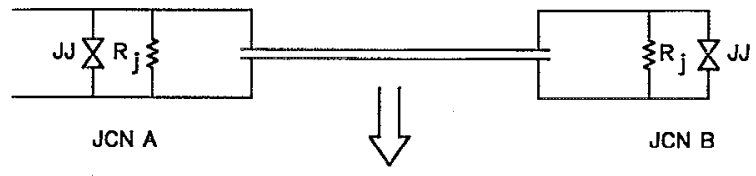

Transmission Line

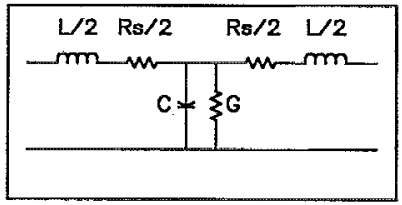

FIG. 8. Two Josephson junctions coupled by a transmission line which is approximated by a series of lumped "T" segments as indicated in the box.

sented a simple load resistor $R_{L}$, must be altered, as shown in Fig. 9, to introduce the required second Josephson junction. This new arrangement is designed to comply with Kirchhoff's law as it applies to the final cell of the linc:

$$
V_{71}-V_{J B}=I_{B} \frac{R_{s}}{2}+\frac{L}{2} \frac{d}{d t}\left(I_{B}\right),
$$

where $I_{B}$ is the net current flowing into junction $B$. As discussed in Ref. 5 , the equivalences between circuit components and transmission line parameters are $C_{c}=C$, $R_{c}=G^{-1}, R^{2} C_{a}=L$, and $R^{2} / R_{a}=R_{s}$. With the particular choices $R=39 \mathrm{k} \Omega, C_{c}=1 \mathrm{nF}, C_{a}=1 \mathrm{nF}, G=(10 \mathrm{M} \Omega)^{-1}$, and $R_{a}=10 \mathrm{M} \Omega$, the equivalents are $C=1 \mathrm{nF}, L=1.52 \mathrm{H}$, $G=10^{-7} \Omega^{-1}$, and $R_{s}=152 \Omega$. The characteristic impedance of such a line is $Z_{0}=\sqrt{ }(L / C)=39 \mathrm{k} \Omega$, and the phase velocity is $v_{\phi}=1 / \sqrt{ }(L C)=25650$ segments per second.

Figure 10 displays the measured applied bias versus junction voltage characteristics. The general shape is essen-

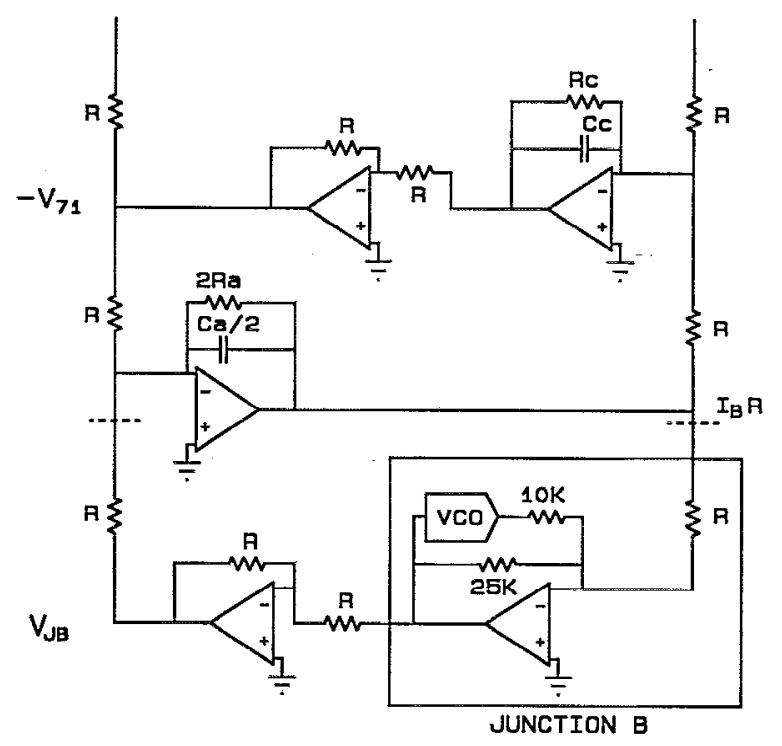

FIG. 9. Schematic of the final section of the analog simulation circuit for the problem of transmission line coupled junctions. The op-amps are type 741 and $R=39 \mathrm{~K}$. 


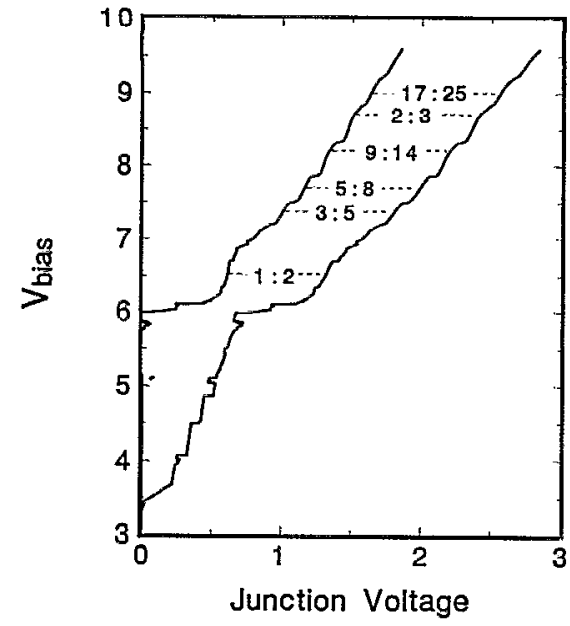

FIG. 10. Input bias (volts) vs time-averaged junction voltage from experiment. Upper characteristic: termination junction; lower characteristic: input junction. Locking ratios for various steps are indicated.

tially the same as the simple resistively coupled case presented in Fig. 3, except that now the curves are "decorated" with additional step structures.

For $V_{\text {bias }}$ between 3.5 and 6.0 , the input junction is in a finite voltage state, but the load junction, which receives less than $I_{c}$, only generates pulses with zero average voltage. Figure 11 shows a typical view of the junction voltage waveforms in this bias interval. These data were obtained by means of a Gould/Biomation model 2805 transient recorder (2048 8-bit samples per channel) which was linked to the GPIB bus with an IO Tech Digital488 interface module. The indicated delay of $2.7 \mathrm{~ms}$ between a given voltage peak at the input junction, and the dynamic response at the load, is simply the propagation time for a signal down the transmission line: $71 / v_{\phi}$. Apart from the
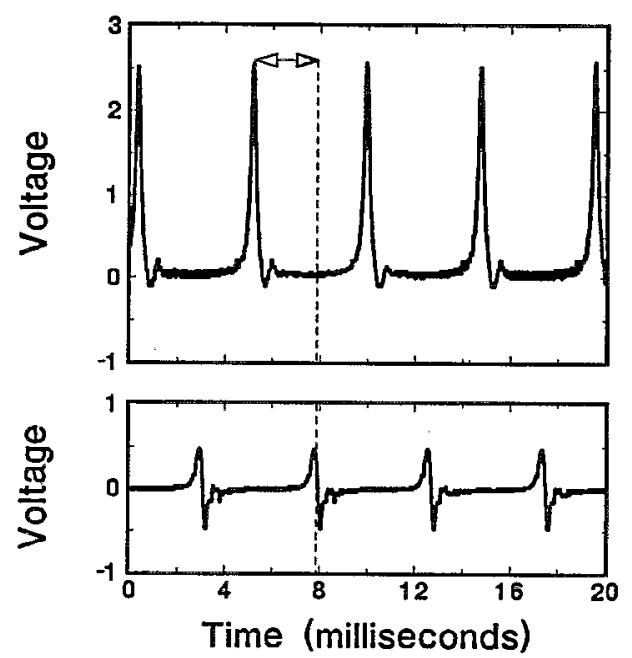

FIG. 11. Junction waveforms at $V_{\text {bias }}=3.8$ from experiment. Upper trace: input junction; lower trace: termination junction. Vertical scales are in volts. The indicated time delay is $2.7 \mathrm{~ms}$. Time is measured from the moment when the transient recorder is triggered.
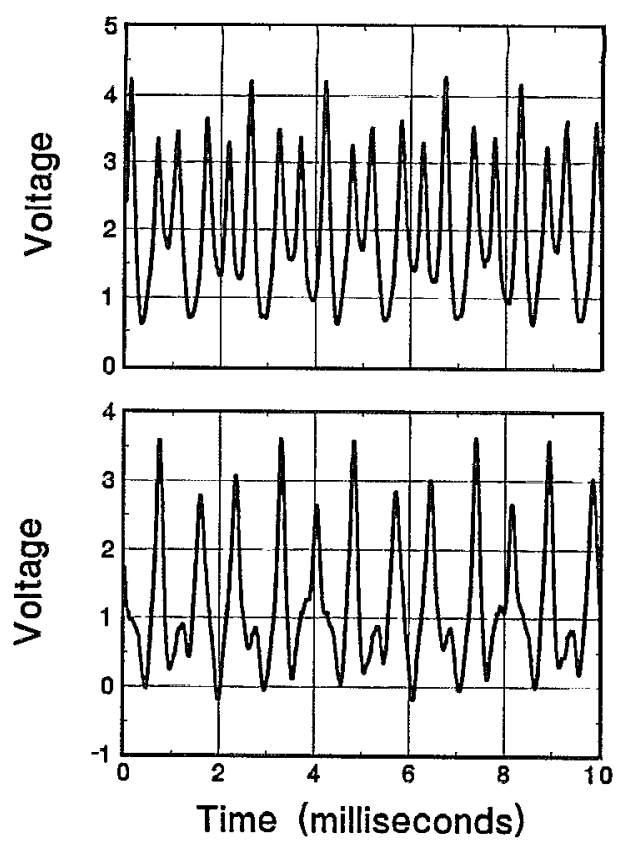

FIG. 12. Junction waveforms at $V_{\text {bias }}=7.7$ from experiment. Upper trace: input junction; lower trace: termination junction. Vertical scales are in volts. The waveforms repeat in $4.14 \mathrm{~ms}$. Time is measured from the moment when the transient recorder is triggered.

delay, one may note the similarity to the corresponding picture for the resistively coupled case, as in Fig. 4.

To lowest order the termination may be viewed as a quasishort in this bias range. The structure on the lower portion of the " $I-V$ " curve of the input junction can then be understood in terms of the resonant steps for a shorted line which were discussed in Refs. 5 and 15.

Our main interest here lies above $V_{\text {bias }}=6.0$ where both junctions develop a dc voltage. A typical set of junction waveforms measured at $V_{\text {bias }}=7.70$ is shown in Fig. 12. The common repeat interval is $4.14 \mathrm{~ms}$, which is equivalent to a frequency of $242 \mathrm{~Hz}$. The power spectra are given in Fig. 13, where it can be seen that the peaks are positioned at increments of $242 \mathrm{~Hz}$. As in the resistively coupled case, there are two principal components, $f_{A}$ and $f_{B}$, together with various sums and differences. In this particular example, the locking ratio is $f_{B}: f_{A}=5: 8$. We have confirmed by integrating the voltage waveforms given in Fig. 12, that, over a period of $4.14 \mathrm{~ms}$, the input junction phase does indeed rotate by eight times $2 \pi$ while the load junction phase rotates by five times $2 \pi$. The various locking ratios which appear as step labels in Fig. 11 were determined by examining the power spectra of $V_{J A}(t)$ and $V_{J B}(t)$ at the relevant bias levels. The pattern of these ratios- 1:2, 3:5, 5:8, 9:14, 2:3, 17:25 forms a monotonic sequence leading towards the limiting value $\alpha /(\alpha+1)=0.698$.

Figure 14 is a magnified view of the 5:8 and 9:14 steps. A number of points were selected along these steps for closer study. At each chosen $V_{\text {bias }}$, the voltage waveforms $V_{J A}(t)$ and $V_{J B}(t)$ were acquired in the transient recorder. The 2048 samples were normally taken at intervals of 50 or 

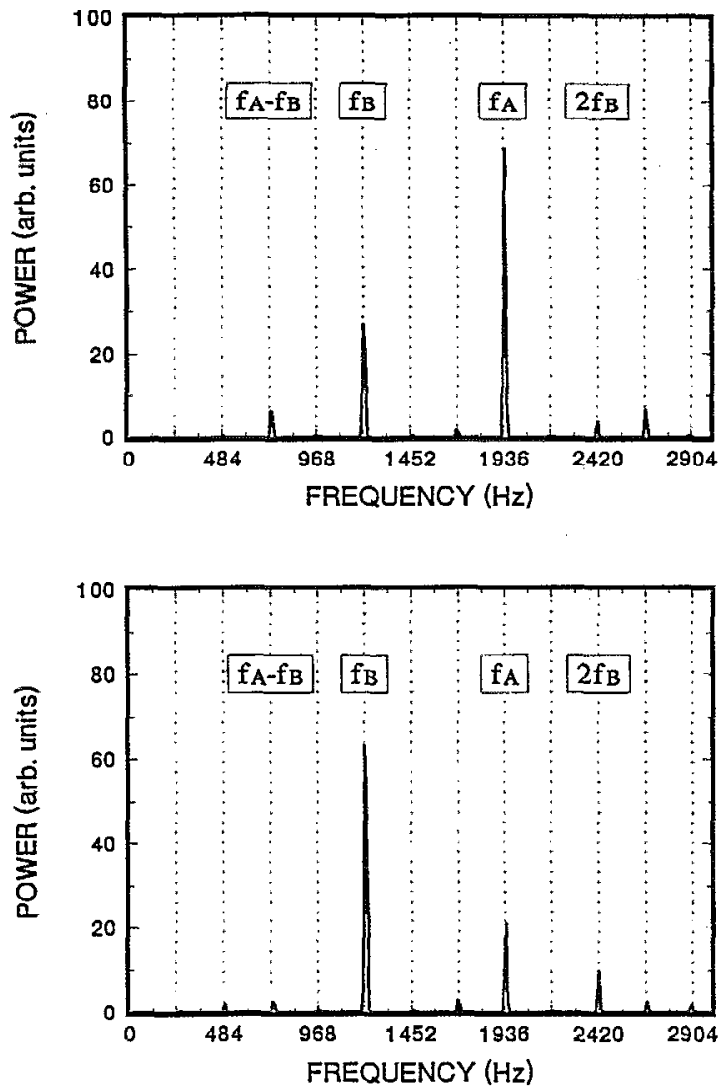

FIG. 13. Power spectra of junction waveforms at $V_{\text {bias }}=7.7$ from experiment. Upper: input junction; lower: termination junction.

$100 \mu$ s. The digitized waveforms were then transferred to the PC and power spectra were computed. From these spectra, as discussed already, the principal components $f_{A}$ and $f_{B}$ were easily found. The resulting data are plotted in Fig. 15. Along each step the dominant frequency is seen to increase linearly with $V_{\text {bias }}$, with a jump to a new linear segment occurring at the top and bottom of the step. It was found from the power spectra that although $f_{A}$ and $f_{B}$

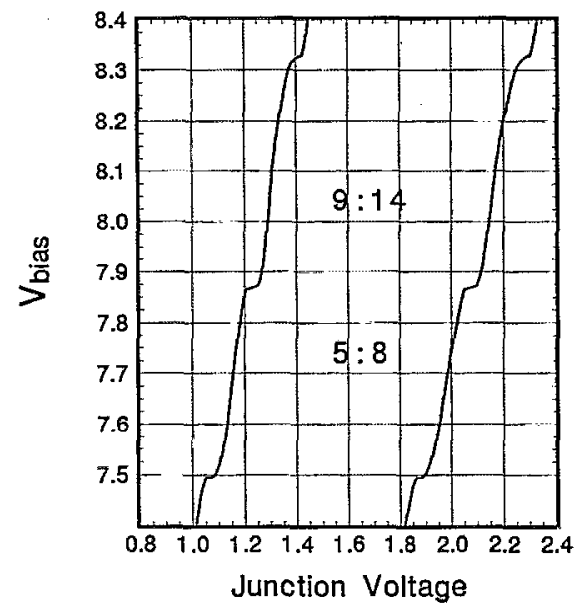

FIG. 14. Expanded portion of characteristics in Fig. 10 showing the 5:8 and 9:14 steps.

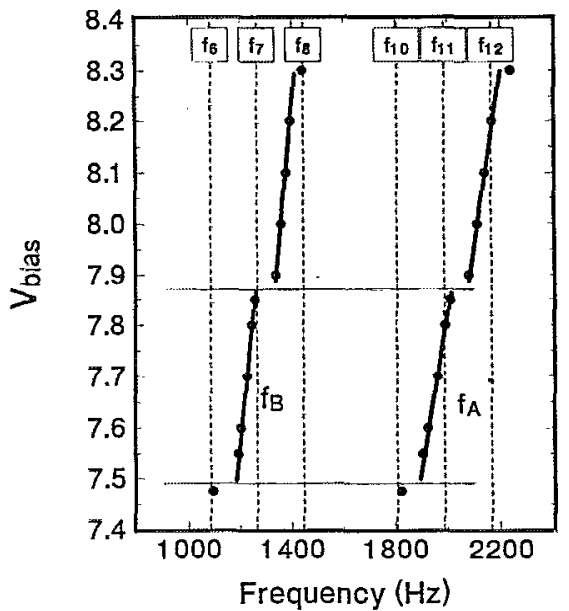

FIG. 15. Principal frequencies $f_{A}$ and $f_{B}$ along the 5:8 and 9:14 steps.

change along any step, they remain locked at the designated ratio for that step. What is different between this situation and the previous resistively coupled case, which has no steps, is that the transmission line resonances are asserting their influence on the system. This is a situation reminiscent of the frequency pulling which occurs when a Josephson junction is placed in a resonant cavity. ${ }^{16}$

The resonant frequencies of a transmission line are associated with the possible standing wave modes. For $n$ standing half waves, they are given by $f_{n}=n\left(v_{\phi} / 2 \times 71\right)$ $=n(180.6) \mathrm{Hz}$. In Fig. 15 it can be seen that the top of the $5: 8$ step occurs at $V_{\text {bias }}=7.87$, where the principal frequency of junction $A$ equals $f_{11}$, and the principal frequency of junction $B$ equals $f_{7}$. Two resonances are simultaneously present in the transmission line, with the input junction acting as the source for one and the terminating junction acting as the source for the other. In this case there are 7 half wavelengths at the frequency of junction $B$ and 11 half wavelengths at the frequency of junction $A$. For lower points on this 5:8 step, simple standing waves do not occur. Here the principal junction frequencies, while not strictly aligned with $f_{11}$ and $f_{7}$, clcarly arc pulled ${ }^{16}$ towards these resonances. Similar effects are evident in the 9:14 step, which is controlled by the $f_{12}$ and $f_{8}$ resonances.

Thus at each step there are two competing phenomena to control the junction frequencies. The first is the resonance condition for which an integer number of standing half waves exists in the transmission line, and the second is the synchronization requirement that the junction frequencies should form a commensurate ratio.

\section{CONCLUSIONS}

The results of these numerical and electronic analog simulations demonstrate that the behavior of a pair of Josephson junctions coupled through a transmission line may be understood in terms of two separate processes. First, the purely resistive link provided by the transmission line enables the junction oscillations to lock so that for any given external bias, the oscillations sharc some common funda- 
mental periodicity. Within this constraint, each junction has a unique waveform whose dominant Fourier component is an integer multiple of the reciprocal of this common period. Second, the reactive properties of the transmission line induce steps in the junction $I-V$ curves. These steps originate in the competition between the transmission line's tendency to resonate, and the junctions' necessity to lock.

\section{ACKNOWLEDGMENT}

This research was supported by grants from the Natural Sciences and Engineering Research Council of Canada.

${ }^{1}$ Superconducting Electronics, edited by H. Weinstock and M. Nisenoff, NATO ASI Series (Springer, Berlin, 1989).

${ }^{2}$ R. L. Kautz, IEEE Trans. Magn. MAG-15, 566 (1979).

${ }^{3}$ J. M. Chwalek, D. R. Dykaar, J. F. Whitaker, R. Sobolewski, S. Gupta,
T. Y. Hsiang, and G. A. Mourou, IEEE Trans. Magn. MAG-25, 814 (1989).

${ }^{4}$ A. D. Smith, B. J. Dalrymple, A. H. Silver, R. W. Simon, and J. F. Burch, IEEE Trans. Magn. MAG-23, 796 (1987).

${ }^{5}$ J. A. Blackburn, H. J. T. Smith, and N. Grønbech-Jensen, J. Appl. Phys. 70, 2395 (1991).

${ }^{6}$ I. I. Blekhman, Synchronization in Science and Technology (ASME, New York, 1988).

${ }^{7}$ M. A. H. Nerenberg, J. A. Blackburn, and D. W. Jillie, Phys. Rev. B 21, 118 (1980).

${ }^{8}$ L. Zhang, Y-D Dai, and Y. H. Kao, J. Appl. Phys. 54, 4494 (1983).

${ }^{9}$ G. S. Lee and S. E. Schwarz, J. Appl. Phys. 60, 485 (1986).

${ }^{10} \mathrm{H}-\mathrm{G}$ Meyer and W. Krech, J. Appl. Phys. 68, 2868 (1990).

${ }^{11}$ P. Hadley and M. R. Beasley, Appl. Phys. Lett. 50, 621 (1987).

${ }^{12}$ Y-D Dai and Y. H. Kao, J. Appl. Phys. 52, 4135 (1981).

${ }^{13}$ B. Wu, Z-J Yang, J. A. Blackburn, S. Vik, H. J. T. Smith, and M. A. H. Nerenberg, Phys. Rev. B 37, 3349 (1988).

${ }^{14}$ A. Barone and G. Paterno, Physics and Applications of the Josephson Effect (Wiley, New York, 1982), p. 127.

${ }^{15}$ N. Gronbech-Jensen, J. A. Blackburn, B. A. Huberman, and H. J. T. Smith, Phys. Lett. A 172, 131 (1992).

${ }^{16}$ B. T. Ulrich, Phys. Lett. A 42, 119 (1972). 